\title{
Algoritmo RSFK para busca de similaridade em GPU
}

\author{
Bruno Henrique Meyer ${ }^{1}$, Wagner M. Nunan Zola ${ }^{1}$, Aurora Trinidad Ramirez Pozo ${ }^{1}$ \\ ${ }^{1}$ Departamento de Informática \\ Universidade Federal do Paraná (UFPR) - Curitiba , PR - Brazil \\ \{bhmeyer, wagner, aurora\}einf.ufpr.br
}

\begin{abstract}
Resumo. Este trabalho apresenta um algoritmo chamado RSFK para computar a estrutura KNN Graph utilizando GPU. Foi possivel observar que o RSFK obteve melhores resultados em comparação às outras opções observadas na literatura, considerando o tradeoff entre tempo e acurácia do resultado.
\end{abstract}

\section{Introdução e Trabalhos Relacionados}

A estrutura que representa os $\mathrm{K}$ vizinhos mais próximos para cada elemento de um conjunto de pontos é definida como K-Nearest Neighbors Graph (KNN Graph), e é utilizada em diversos cenários, incluindo algoritmos de aprendizado de máquina [Tang et al. 2016]. Devido à natureza da complexidade quadrática do tempo necessário para computar o KNN Graph, diversos algoritmos foram propostos para aproximar a busca dos vizinhos [Yan et al. 2019, Johnson et al. 2019]. No algoritmo Random Projection Forest KNN (RPF-KNN) [Yan et al. 2019], as partições são criadas por meio da projeção dos pontos em um hiperplano aleatório. Após a projeção, os pontos estarão ordenados e poderão ser separados em duas partições de tamanho equivalente. A partir delas o mesmo processo poderá ser realizado recursivamente, o que pode ser representado por uma árvore binária. Neste trabalho, apresentamos uma variante do RPF-KNN chamada Random Sample Forest KNN, que iremos abreviar como RSFK. No RSFK, a mesma estratégia do RPF-KNN é utilizada, com a diferença de que a criação de um hiperplano aleatório e a projeção dos pontos não são etapas necessárias. Na nossa abordagem, são gerados hiperplanos equidistantes a dois pontos da partição, que são selecionados aleatoriamente. As primitivas cuRAND da linguagem CUDA são utilizadas para gerar números pseudoaleatórios. Dessa forma, o lado em que cada ponto ficará em relação ao hiperplano será usado como critério para definir quais serão as novas partições.

Na biblioteca ANNOY, que implementa o RPF-KNN, a estratégia para paralelizar o algoritmo consiste em criar uma thread da CPU para computar cada árvore, o que é adequado apenas quando poucos núcleos de processamento serão utilizados, o que não é o caso de arquiteturas de GPU. A nossa abordagem permite realizar a paralelização criando uma árvore por vez, porém de forma paralelizada inteiramente em GPU ao criar uma thread da GPU para cada ponto. Para implementar esta abordagem, é necessário utilizar operações atômicas para garantir a sincronização de todas as threads. Para implementar o RSFK, também possibilitamos o uso da técnica Nearest Neighbor Exploring (NN Exploring) [Tang et al. 2016], uma abordagem para o pós-processamento dos dados para melhorar a acurácia da aproximação.

\section{Materiais e Métodos}

Testamos nossa implementação do RSFK em GPU com processador i5-4460 3.20GHz (4 núcleos) com GPU NVIDIA RTX 2070 e CUDA 10.1. Nos experimentos foram utilizadas as bases de dados MNIST e ImageNet. O KNN Graph de cada base foi computado 
usando as bibliotecas FAISS [Johnson et al. 2019], e ANNOY, e a nossa implementação RSFK. Cada técnica foi executada diversas vezes alterando os parâmetros que controlam o tradeoff entre tempo e acurácia O RSFK teve 2 execuções adicionais para avaliar a estratégia NN Exploring. A acurácia do RSFK e ANNOY é controlada pelo número de árvores geradas, que são combinadas (ensemble) para obter um KNN Graph mais preciso. Em nossos experimentos, variamos o número de árvores entre 1 e 896 para o RSFK, e entre 1 e 21 para o ANNOY. Cada árvore pode ser considerada uma execução do algoritmo (cada árvore usa uma semente diferente para os geradores de números pseudoaleatórios). $\mathrm{Na}$ biblioteca FAISS, podemos controlar a acurácia do método por meio do parâmetro nprobe, que foi variado entre 1 e 20. Para cada execução, consideramos o número de vizinhos $(\mathrm{K})$ igual a 32. Para cada técnica, medimos o número médio de pontos processados por segundo e a acurácia da aproximação do KNN-Graph em comparação com o seu resultado exato (porcentagem de vizinhos corretos).

\section{Resultados e Conclusões}

Na Figura 1 identificamos que o RSFK obteve o melhor tradeoff dentre as técnicas comparadas. Verificamos que o uso da estatégia NN Exploring apresentou uma melhora apenas quando é necessário criar o KNN Graph com acurácias próximas de 100\%. Concluímos que as evidências apresentadas neste trabalho indicam que o algoritmo RSFK pode trazer vantagens em acurácia e/ou tempo em relação à biblioteca ANNOY e ao método IVFLAT da biblioteca FAISS. Em trabalhos futuros pretendemos aplicar o algoritmo no contexto de aplicações em Aprendizado de Máquina, por exemplo na redução de dimensionalidade [Meyer et al. 2020] para visualização e interpretação de grandes datasets.
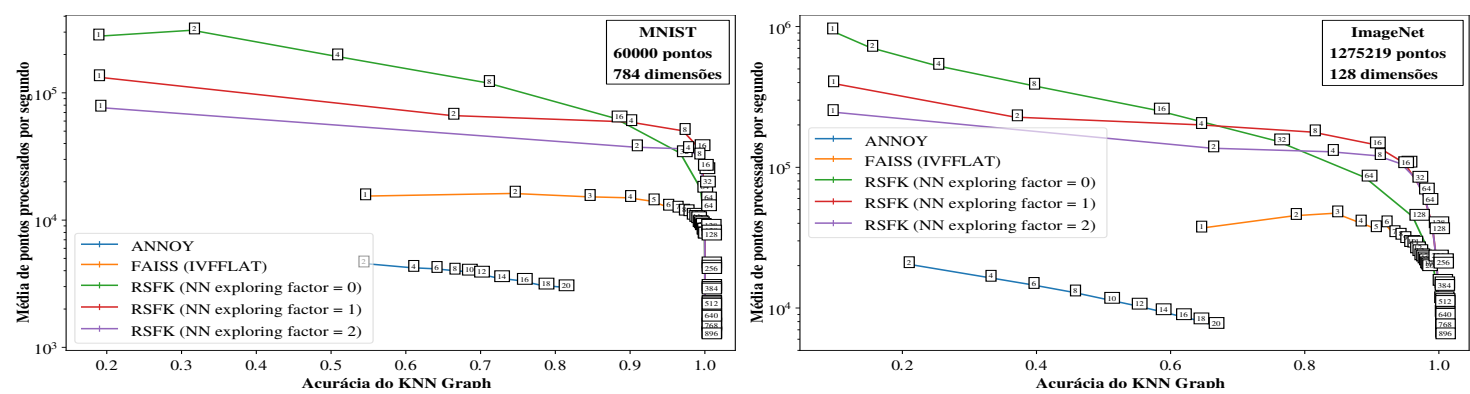

Figura 1. Comparação de algoritmos . Cada ponto representa a execução de um algoritmo com uma variação de parâmetro, nprobe para o FAISS (IVFFLAT) e número de árvores para ANNOY e RSFK.

\section{Referências}

Johnson, J., Douze, M., and Jégou, H. (2019). Billion-scale similarity search with GPUs. IEEE Transactions on Big Data.

Meyer, B. H., Pozo, A. T. R., and Zola, W. M. N. (2020). Improving Barnes-Hut t-SNE scalability in GPU with efficient memory access strategies. In 2020 International Joint Conference on Neural Networks (IJCNN).

Tang, J., Liu, J., Zhang, M., and Mei, Q. (2016). Visualizing large-scale and highdimensional data. 25th International World Wide Web Conference, WWW 2016.

Yan, D., Wang, Y., Wang, J., Wang, H., and Li, Z. (2019). K-nearest Neighbors Search by Random Projection Forests. IEEE Transactions on Big Data, 7790. 